\title{
Medical Image Compression Using Multiwavelet Transform
}

\author{
N.Thilagavathi ${ }^{1}$, K.Subramani ${ }^{2}$ \\ ${ }^{1} P G$ Scholar-M.E. (Applied Electronics) \\ Hindusthan College of Engineering and Technology \\ Coimbatore - 641032. \\ ${ }^{2}$ Asst.Prof / Electrical and Electronics Engineering \\ Hindusthan College of Engineering and Technology \\ Coimbatore-641032.
}

\begin{abstract}
With the development of micro electronics and information technology, there are more pixels in unit area of the imaging device. The high resolution images with more pixels can be obtained easily. But the high resolution images have huge data volume. It is difficult to transmit, to process, to store high resolution images. So compressing images is an important technology to reduce the requisite storage space and transmission channel bandwidth. In this paper multiwavelet coding has been proved to be a very effective technique for medical images giving significantly better results. The Discrete Multiwavelet Transform of the image is calculated with Geronimo- Hardin - Massopust (GHM) multiwavelet. The wavelet coefficients are encoded using SPIHT coder. This method yields better compression performance. The quality of the image is assessed by PSNR value, Compression Ratio and Coding times.
\end{abstract}

Keywords - DiscreteMultiwaveletTransform(DMWT), Geronimo- Hardin - Massopust (GHM) multiwavelet, Good Multifilter Propertity (GMP), Set Partitioning In Hierarchical Trees (SPIHT), Peak Signal to Noise Ratio (PSNR), Compression Ratio (CR), Coding times(Encoding and Decoding times).

\section{INTRODUCTION}

High-Performance compression methodologies for the transmission of medical data and images play an important part in high-speed, cost-effective, and efficient networked telemedicine services. However, the recent developments of commercial compression tools and technologies for multimedia, Internet, and other applications, are not paralleled with similar work on application-specific compression methodologies for telemedical systems and, in particular, for wireless telemedicine applications. Multiwavelet compression has been developed by many authors $[1,2]$. In recent years, multiwavelet-based compression techniques and tools have received significant attention, especially for different biomedical signal-processing applications. The objective of image compression techniques is to eliminate redundant data present in image such that it allows for satisfactory image reconstruction. Generally in image compression, an original image mapped onto a bit stream appropriate for communication over or storage in a digital medium. Obviously, the number of bits needed to represent the coded image should be lesser than that required for the original image.

In image compression, multiwavelets can be defined as mathematical functions that offer very good quality compression at very high ratios owing to their capability to decompose signals into different scales or resolutions. In general, multiwavelets can possess all the desirable properties important in signal processing and image processing, such as orthogonality, compact support,linear phase and high approximation/vanishing moments of the basis function, efficient multi-resolution representation, scalability, and embedded coding with progressive transmission. The general procedure involved in multiwavelet transform-based image compression techniques is, first the image data is decorrelated by applying a multiwavelet transform, then the resulting transform coefficients are quantized and the quantized values are coded. Of most algorithms developed, SPIHT algorithm ever since its introduction in 1996 for image compression has received a lot of attention.

For medical image compression, SPIHT achieves considerably better quality when compared to Vector quantization, JPEG, and Wavelets combined with quantization techniques, while not requiring training and producing an embedded bit stream. Primarily, SPIHT is a wavelet-based image compression coder; multiwavelet transform has been made use of for image decomposition. The performance of the proposed scheme is evaluated in terms of PSNR and the Compression Ratio attained. The experimental results demonstrate the effectiveness of the proposed scheme in image compression. A major design goal of any compression method is to obtain the visual quality with lowest bit rate. However, the quality and the bit rate are the trade-offs that must be considered simultaneously. 
The rest of the paper is organized as follows. Section II presents an overview of multiwavelets. Section III discusses the SPIHT coder. Section IV presents the proposed coding method. Section V shows experimental results of the proposed work. Section VI gives some concluding remarks.

\section{MULTIWAVELETS}

Multiwavelets can be considered as vector-valued wavelets [3], which satisfy conditions in which matrices are involved, rather than scalars, as in the single wavelets. This is an advantage for the image classification, since it is possible to have a larger number of sub bands and better energy compaction capability with multiwavelets than with scalar wavelets [4]. In 1988, Mallat proposed the concept of "multi-resolution analysis" [5], the wavelet transform is used in signal processing, the algorithm and reconstruction thought of the signal and image resolution for different frequency channel are given [6], which makes wavelet transform been used in the signal processing and image processing, voice analysis, pattern recognition, communication, earthquake, computer vision and aspects of quantum physics. In multiresolution analysis of multiplicity $r$, the multiscaling functions and the corresponding multiwavelets are usually written as vectors $\phi(t)$ and $\psi(t)$, satisfying the matrix dilation equation

$$
\begin{gathered}
\varphi(t)=\sqrt{2} \sum_{k=-\infty}^{k k=\infty} H_{k} \varphi(2 t-k) \\
\Psi(t)=\sqrt{2} \sum_{k=-\infty}^{k=\infty} G_{k} \varphi(2 t-k)
\end{gathered}
$$

where $\mathrm{Hk}$ and Gk are the lowpass and highpass filter coefficients respectively. Unlike scalar wavelets, in which Mallat's pyramid algorithms can be employed directly, the application of multiwavelets requires, the input signal to be first vectorized (which is a problem popularly known as multiwavelet pre-filtering). The decomposition of the multiwavelet transformation for 2-dimensional images is carried out using tensor products of the 1-dimensional filter banks as illustrated in figure1.

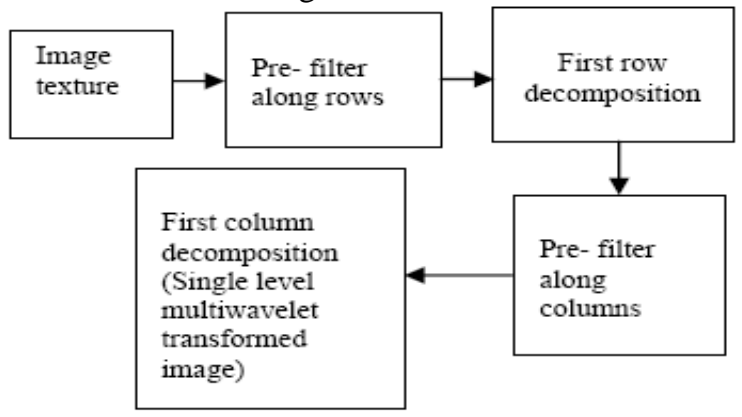

Figure 1: Decomposition Process of multiwavelet transformation

The single level decomposition of the multiwavelet transformation produces 16 sub bands, as illustrated figure2.

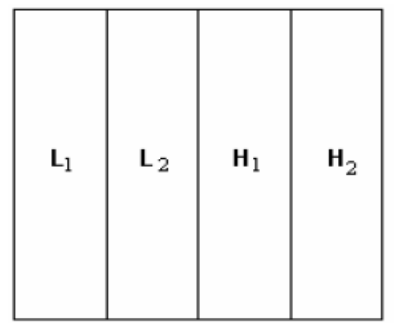

(a)

\begin{tabular}{|l|l|l|l|}
\hline $\mathrm{L}_{1} \mathrm{~L}_{1}$ & $\mathrm{~L}_{1} \mathrm{~L}_{2}$ & $\mathrm{~L}_{1} \mathrm{H}_{1}$ & $\mathrm{~L}_{1} \mathrm{H}_{2}$ \\
\hline $\mathrm{L}_{2} \mathrm{~L}_{1}$ & $\mathrm{~L}_{2} \mathrm{~L}_{2}$ & $\mathrm{~L}_{2} \mathrm{H}_{1}$ & $\mathrm{~L}_{2} \mathrm{H}_{2}$ \\
\hline $\mathrm{H}_{1} \mathrm{~L}_{1}$ & $\mathrm{H}_{1} \mathrm{~L}_{2}$ & $\mathrm{H}_{1} \mathrm{H}_{1}$ & $\mathrm{H}_{1} \mathrm{H}_{2}$ \\
\hline $\mathrm{H}_{2} \mathrm{~L}_{1}$ & $\mathrm{H}_{2} \mathrm{~L}_{2}$ & $\mathrm{H}_{2} \mathrm{H}_{1}$ & $\mathrm{H}_{2} \mathrm{H}_{2}$ \\
\hline
\end{tabular}

(b)

Figure 2: Image sub band structure for first level of decomposition. (a)Filtering along Horizontal direction. (b)Filtering along Vertical direction

Here a typical block H2L1 contains low pass coefficients corresponding to the first scaling function in the horizontal direction, and high pass coefficients corresponding to the second wavelet in the vertical direction. 


\section{SPIHT CODER}

SPIHT is one of the most advanced schemes available that outperforms even the state-of-the-art JPEG 2000 in some situations. Said and A. Pearlman developed a SPIHT coding algorithm [7], a refined version of embedded zero tree wavelet coder (EZW) coder [8]. The Set-Partitioning in Hierarchical Trees (SPIHT) coding operates by exploiting the relationships among the multiwavelet coefficients across the different scales at the same spatial location in the wavelet sub bands. The SPIHT method is not a simple extension of traditional methods for image compression, and represents an important advance in the field. The method deserves special attention because provides the following: highest image quality, progressive image transmission, fully embedded coded file, simple quantization algorithm, fast coding/decoding, completely adaptive, lossless compression, exact bit rate coding, and error protection.

In general, SPIHT coding involves the coding of the position of significant multiwavelet coefficients and the coding of the position of zero trees in the multiwavelet sub bands. The SPIHT coder exploits the following image characteristics: 1) the majority of an image's energy is concentrated in the low frequency components and a decrease in variance is observed when move from the highest to the lowest levels of the sub band pyramid and 2) it has been observed that there is a spatial self-similarity among the sub bands, and the coefficients are likely to be better magnitude-ordered if it moves downward in the pyramid along the same spatial orientation. A tree structure, termed spatial orientation tree, clearly describes the spatial relationship on the hierarchical pyramid. Figure3 shows how the spatial orientation tree is defined by a Parent-children relationship among the 4 subbands. Every pixel in the image signifies a node in the tree and is determined by its corresponding pixel coordinate. Its direct descendants (offspring) symbolize the pixels of the same spatial orientation in the next finer level of the pyramid. The tree is defined in such a manner that each node has either no offspring (the leaves) or four offspring's, which at all times form a group of $2 \times 2$ adjacent pixels. In figure 3 , the arrows are directed from the parent node to its four offspring's. The pixels in the highest level of the pyramid are the tree roots and are also grouped in $2 \times 2$ adjacent pixels. Nevertheless, their offspring branching rule is different, and in each group, one of them has no descendants.

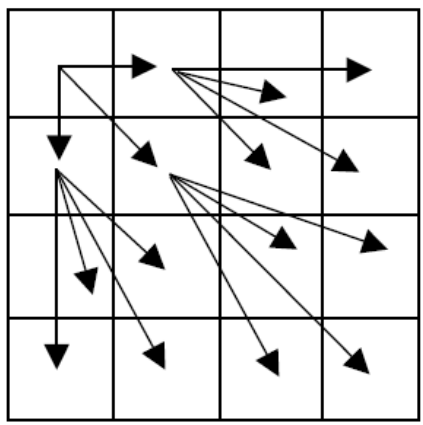

Figure 3: Parent-children relationship among the 4 subbands.

SPIHT is based on three concepts (1) exploitation of the hierarchical structure of the wavelet transform by using tree-based organization of the coefficients, (2) partial ordering of the transformed coefficients by magnitude, (3) ordered bit plane transmission of refinement bits for the coefficient values. This leads to a compressed bit stream in which the most important coefficients are transmitted first. The values of all coefficients are progressive refined and the relationship between coefficients representing the same location at different scales is fully exploited for compression efficiency. The SPIHT algorithm appears to give extremely good performance in digital image compression. The fully embedded nature of the output bit stream also makes an excellent choice for progressive transmission. The interband spatial dependencies are captured in the form of parent-child relationships. With the exception of the coarsest subband and the finest subbands each wavelet coefficient at the $\mathrm{i}$-th level of composition is spatially correlated to 4 child coefficients at level i- 1 in the form of $2 \times 2$ blocks of adjacent pixels. These 4 child coefficients are at the same relative location in the subband decomposition structure. This relationship is utilized during SPIHT quantization. If a parent coefficient is insignificant with respect to a particular threshold then all of its children would most likely be insignificant and similarly significant coefficients in the finer subband most likely correspond to a significant parent in the coarser subband. This result in the significant savings: only the parent's position information needs to be coded since the children's coordinate scan is inferred from the parent's position information. 


\section{THE PROPOSED CODING METHOD}

The block diagram of the proposed method is shown in figure 4. Medical images are first decomposed using GHM multiwavelet and the multiwavelet coefficients are encoded using SPIHT. The algorithm starts at the coarsest sub band in the sub band pyramid. SPIHT captures the current bitplane information of all the DMWT coefficients and organizes them into three subsets: (1) List of Significant Pixels (LSP), (2) List of Insignificant Pixels (LIP) and (3) List of Insignificant Sets of Pixels (LIS)). LSP constitutes the coordinates of all coefficients that are significant. LIS contains the roots of insignificant sets of coefficient. Finally, LIP contains a list of all coefficients that do not belong to either LIS or LSP and are insignificant. During the encoding process these subsets are examined and labeled significant if any of its coefficients has a magnitude larger than a given threshold. The significance map encoding (set partitioning and ordering pass) is followed by a refinement pass, in which the representation of significant coefficients is refined. The thresholds used to test significance are powers of two, so in its essence, the SPIHT algorithm sends the binary representation of the integer value of multiwavelet coefficients.

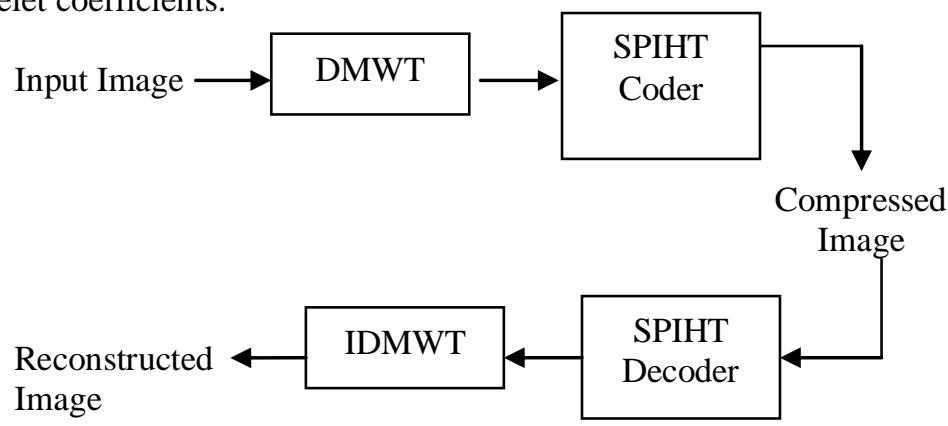

Figure 4: Block Diagram of Proposed Coding Method

\section{EXPERIMENTAL RESULTS}

Medical images of resolution 128 x 128pixels, 256 x 256 pixels and 512 x 512 pixels may be used for experiments. The image is first decomposed using GHM multiwavelet filter and the multiwavelet coefficients are encoded using SPIHT coder and compressed bit stream is sent to the decoder. The original and reconstructed images of various bit rates and decomposition level of 2 , processed by this algorithm is shown in figure 5 . The reconstructed image at a low bit rate shows good quality without distortion. The effectiveness of the algorithm described above can be statistically modeled and evaluated.

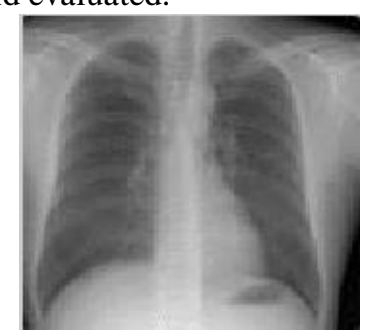

Figure 4(a): Input Image: Chest.jpg, Size: 128 x128 pixels, No. of bits: 131072bits.
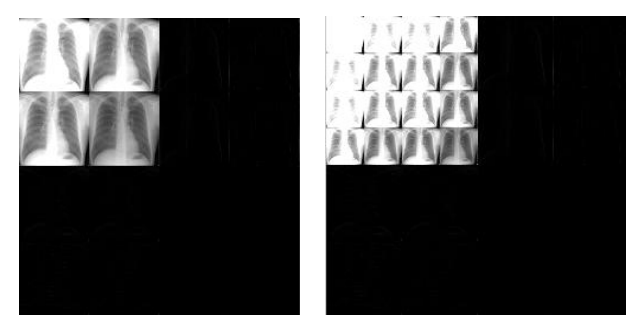

Figure 4(b): Single level and two level decomposed images through GHM multiwavelet 


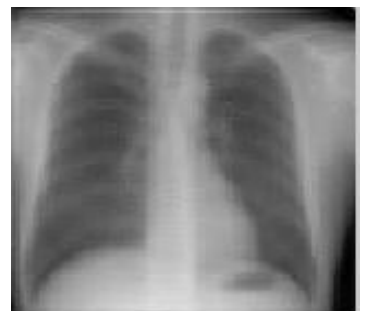

Figure 4(c) Reconstructed Image of PSNR=38.85dB at $0.5 \mathrm{bpp}$

The performance analysis of the algorithm is measured in terms of MSE, PSNR, and CR calculations and which are defined in equations (3), (4), and (5) respectively.

$$
M S E=\frac{1}{m n} \sum_{i=0}^{m-1} \sum_{j=0}^{n-1}\lfloor I(i, j)-K(i, j)\rfloor^{2}
$$

where, $m$ is the no. of rows, $n$ is the no. of columns, $I(i, j)$ is the original image and $K(i, j)$ is the reconstructed image.

$$
P S N R=20 \log _{10}\left(\frac{M A X_{I}}{\sqrt{M S E}}\right)
$$

where, MAXI is the maximum possible pixel value of the image.

$$
\text { Compression Ratio }(C R)=\frac{\text { Original Size }}{\text { Compressed Size }}
$$

(5)

The objective quality measures correlate with the perceived image quality and the numerical results are shown in Table I.

TABLE I. NUMERICAL RESULTS

\begin{tabular}{|c|c|c|c|c|}
\hline $\begin{array}{c}\text { Compression } \\
\text { Analysis } \\
\text { Parameters }\end{array}$ & \multicolumn{4}{|c|}{$\begin{array}{c}\text { Results of Proposed Algorithm at } \\
\text { various Bit Rates (bpp) }\end{array}$} \\
\cline { 2 - 5 } & 1 & 0.5 & 0.25 & 0.125 \\
\hline PSNR (dB) & 38.86 & 38.85 & 38.84 & 35.86 \\
\hline $\begin{array}{c}\text { Compression } \\
\text { Ratio }\end{array}$ & $128: 1$ & $64: 1$ & $32: 1$ & $16: 1$ \\
\hline No. Of Bits & 16384 & 8192 & 4096 & 2048 \\
\hline $\begin{array}{c}\text { Encoding Time } \\
\text { (sec) }\end{array}$ & 56.45 & 51.11 & 25.30 & 7.84 \\
\hline $\begin{array}{c}\text { Decoding Time } \\
\text { (sec) }\end{array}$ & 49.00 & 46.23 & 19.02 & 4.44 \\
\hline
\end{tabular}

\section{CONCLUSION}

A new method for Medical Image Compression through Multiwavelet Transform has been proposed. The method is key for performing effective discrete multiwavelet decomposition and reconstruction algorithms. The pre-filtering work does not only require very low processing overhead but it is also robust enough to work well with any multiwavelets. Experimental results in image compression provided conclusive evidence that the proposed multiwavelets with GMPs could consistently outperform popular scalar wavelets with low computation complexity. We used the jpg, bmp images to test the performance of our proposed algorithm. We used the compression ratio, encoding time, decoding time and PSNR parameters to measure the performance and quality of the reconstructed image. The experiment results are based on Geronimo- Hardin- Massopust (GHM) multiwavelet transform and shows that we can get a good quality of recovered image. And these results also verified the usefulness of multiwavelets for medical image compression. 


\section{REFERENCES}

[1] S. Esakkirajan, T. Veerakumar, V. Senthil Murugan, P. Navaneethan (Aug 2008), "Image Compression Using Multiwavelet and Multi-stage Vector Quantization”, World Academy of Science, Engineering and Technology 48.

[2] Hongxia Ni and Yufeng Li, (Dec.2009), "Study on Compression Method for Noisy image in Wavelet Domain", International Conference on Information Engineering and Computer Science, Pages: 1 -4.

[3] Jo Yew Tham, Lixin Shen, Seng Luan Lee, and Hwee Huat Tan (Feb.2000), “A General Approach for Analysis and Application of Discrete Multiwavelet Transforms”, IEEE Transactions On Signal Processing, Vol. 48, No. 2.

[4] Jo Yew Tham, et.al, (2003), “A New Multifilter Design Property for Multiwavelet Image Compression”, IEEE International Conference on Acoustics, Speech and Signal Processing, Pages: 1229-1232, Vol.3.

[5] Li Xiaoping, Qu Dacheng, Multimedia network communication [MlBeijing: Beijing university of science and technology press 2001.1

[6] Ren Zhengyun Multimedia computer technology [M]Beijing: China water conservancy and hydropower press 2009.7

[7] Said A.Pearlman WA,"A new fast and efficient image codec based on set partitioning in hierarchical trees, "IEEE Trans. Circuits Syst.Video Technology, vol.6, pp. 243-250, June 1996.

[8] Shapiro JM,"Embedded image coding using zero trees of wavelet coefficients, ”IEEE Trans Signal Processing. 41:3445-3462, 1993

\section{BIBLIOGRAPHY}

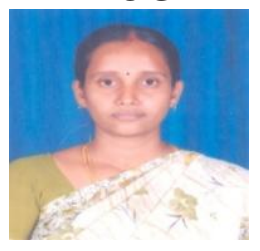

Mrs. N. Thilagavathi was born on April $10^{\text {th }}, 1983$ in Coimbatore, Tamilnadu. She has received her Bachelor of Engineering in the branch of Electronics and Instrumentation at Kongu Engineering College, Perundrai, Tamilnadu in the year 2004

She has worked as a lecturer in Nachimuthu Polytechnic College, Pollachi from the year 2005 to 2010. Now she is doing her Master of Engineering in Hindustan College of Engineering and Technology, under Anna University of Technology, Chennai

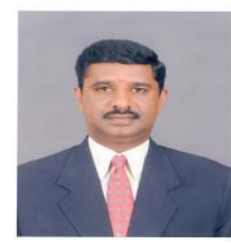

Mr. K. Subramani was born on February $16^{\text {th }}$, 1974 in Coimbatore, Tamilnadu. He has got his Bachelor of Engineering in the branch of Electricals and Electronics at Coimbatore Institute of Technology, Coimbatore, Tamilnadu in the year 2005. He has finished his Master of Engineering degree in Power Electronics and Drives at Bannari Amman Institute of Technology, Sathy, Tamilnadu.

$\mathrm{He}$ is working as an Assistant Professor in Hindustan College of Engineering and Technology, Coimbatore for the past 5 years. His interested areas are Power Electronics and Electric Drives and Image processing. 